\title{
Nasal Smear Eosinophil CountVs AEC of Patients diagnosed with Allergic Rhinitis Attending a Tertiary Care Hospital \&the Effect of Treatment with a TopicalSteroid Nasal Spray on theSame
}

\author{
Dr. Shreepad S Shetty ${ }^{1}$, Dr.Sheetal Rai ${ }^{1}$,Dr Gangadhara Somayaji k s s , Dr. Ali \\ Zameel $^{2}$ \\ ${ }^{I}$ Dept. of ENT, Yenepoya Medical College \& Hospital, Mangalore, India \\ ${ }^{2}$ Dept. of Community Medicine, K.S Hegde Medical Academy, Mangalore, India
}

\begin{abstract}
Allergic rhinitis being a heterogeneous disorderis often under-diagnosed despite its high prevalence. The age, sex, race or socioeconomic status has no bearing on those who get affected.It is one of the most common chronic conditions in the developed world with a significant impact on the quality of life.Methodology: All the patients who were diagnosed with allergic rhinitis satisfying the inclusion criteria were included in the study by universal sampling, after obtaining informed consent. A total of 42 patients were thus included. Statistical Analysis:The baseline data was expressed as percentages \& proportions. Mcnemar Chi square test $\&$ Paired t-test were applied for comparison between the two tests \& for analyzing the pre-treatment \& posttreatment values ofnasal smear eosinophil counts \& AEC. Results: Sensitivities of the tests were calculated as $40 \%$ \& $54 \%$ for nasal smear eosinophil count \& AEC respectively. The difference in sensitivities were found to be significant $(p<0.05)$. The difference in the pre-treatment \& post-treatment values of both nasal smear \& AEC were found to be significant $(p<0.05)$. AEC reduction was observed in $85.7 \%$ of the patients from the pretreatment values \& reduction in the nasal smear eosinophil counts were found in $28.6 \%$ of the patients.
\end{abstract}

Key words:AEC, Allergic rhinitis, Fluticasone nasal spray, Nasal smear eosinophil count

\section{Introduction}

Allergic rhinitis being a heterogeneousdisorder is often under-diagnosed despite its high prevalence.[1] The age, sex, race or socioeconomic status has no bearing with those who get affected.[2]It is one of the most common chronic conditions in thedeveloped world with a significant impact on the quality of life.[3]

The head and neck being the most common portal of entry of inhalants and food sensitizersisthe region where the most common allergy induced diseases, such rhinitis, sinusitis, otitis media, laryngitis and conjunctivitis occurs.[4,5] As the nose is usually the first site of hypersensitivity response the ENT department is frequently called upon to evaluate.[2]Most of the patients suffering from allergic rhinitis can be diagnosed by a combination of thehistory, clinical examination, skin prick test, radio-allegrosorbent assay for specific IgE levels and nasal smear for eosinophils.[5,6]Hence this study was conducted to evaluate the value of nasal smear eosinophil count as a simple non-invasive \& inexpensive method for diagnosing allergic rhinitis \& the effect of flutoicasone nasal spray on local \& systemic eosinophil counts

\section{Methodology}

The study was conducted at our hospital over a period of 6 months from October 2012 to March 2013. The study was initiated after obtaining ethical clearance from the Institutional Ethics Committee. All the patients who were diagnosed with allergic rhinitis satisfying the inclusion criteria were included in the study by universal sampling, after obtaining informed consent. A total of 42 patients were thus included.

The patients were diagnosed purely based on the history \& clinical features typical of allergic rhinitis which included sneezing, rhinorrhoea, nasal itching, nasal obstruction \& pale mucosa.

\subsection{Inclusion Criteria}

i.Patients who have given consent to be a part of the study

ii. Patients above 12 years of age with allergic symptoms

iii. Patients who were not treated with topical steroid nasal spray in the past

\subsection{Exclusion Criteria}

i. All patients who did not give consent to be a part of the study

ii. Patients below 12 years of age

iii. Patients with a history of acute respiratory tract infections in the past month

iv. Snuff users and pregnant women 
v. Patients who were prescribed anti-histaminics within the past week

Following inclusion into the study, nasal smear eosinophil count\& blood AEC (Absolute Eosinophil Count) were performed on all the patients. Nasal smear was performed by scraping the mucous membrane over the inferior turbinate using a sterile air dried cotton applicator \& transferred to a glass slide. The slide was stained with May-Grunwald\&Giemsa stain. AEC was performed on venous blood drawn from the patients' arm using standard procedure. Both the tests were performed by a trained pathologist $\&$ the slides for both the tests were reviewed randomly by another trained pathologist.

The patients were then prescribed with Fluticasone Propionate nasal spray for a month. They were advised to deliver 1 spray to each nostril twice daily (total measuring 200 micrograms/day) for a month. They were asked to return for follow up after a month \& were advised to stop the medication 3 days prior to their return. On follow up the patients were assessed for signs \& symptoms and nasal smear eosinophil count\& AEC were also repeated.

\subsection{Statistical Analysis}

The data was entered into excel, cleaned, coded \& transferred to SPSS. Statistical analysis was performed using SPSS V.16. The baseline data was expressed as percentages \& proportions. McNemar Chi square test \& Paired t-test were applied for comparison between the two tests \& for analyzing the pre-treatment $\&$ post-treatment values of nasal smear eosinophil counts \& AEC.

\section{Results}

Out of the 42 patients in this study, $57.1 \%$ of the patients presented with history of seasonal allergy, $19 \%$ presented with history of perennial allergy and the remaining $23.9 \%$ presented with mixed symptoms (i.e. both seasonal and perennial)

Figure 1: Age distribution of patients with Allergic Rhinitis

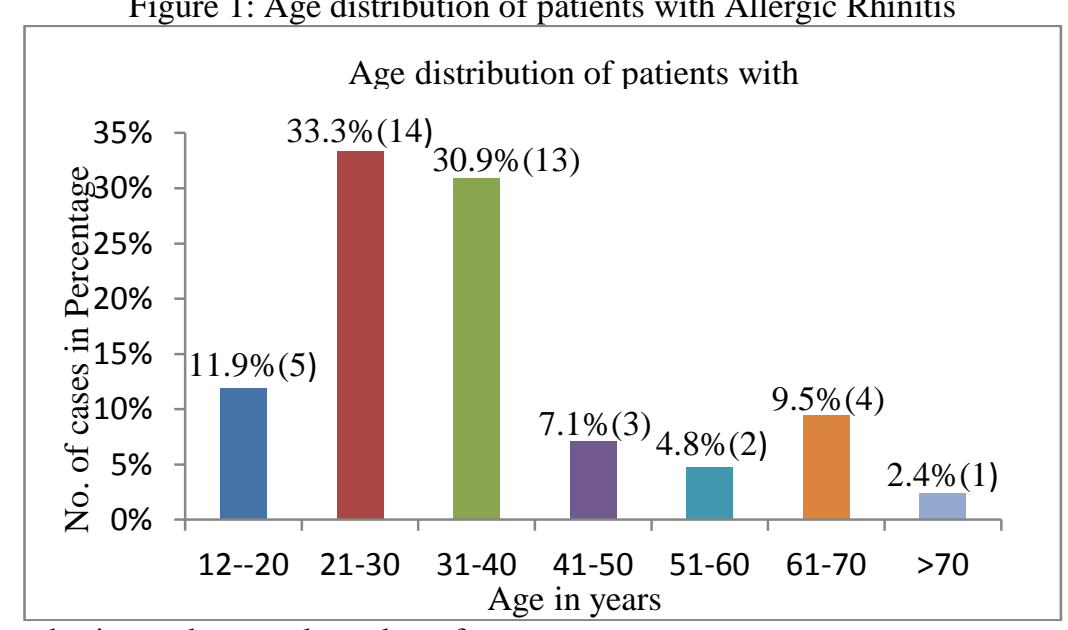

The figures in parenthesis are the actual number of cases

Majority of the patients belonged to the age group of 20-40 years(64.2\%)

Table 1: Sex Incidence of Allergic rhinitis among the patients

$\mathrm{M}: \mathrm{F}$ ratio $=1.6: 1$

\begin{tabular}{|c|c|c|}
\hline Sex & Frequency $(\mathrm{n})$ & Percent \\
\hline $\mathrm{M}$ & 26 & 61.9 \\
\hline $\mathrm{F}$ & 16 & 38.1 \\
\hline Total & 42 & 100 \\
\hline
\end{tabular}

The incidence of allergic symptoms was more common among males (61.9\%). Male:Female ratio was 1.6:1

Table 2: Frequency of signs \& symptoms

\begin{tabular}{|l|l|l|}
\hline Signs \& Symptoms & Frequency & Percent \\
\hline Rhinorrhoea & 41 & 97.6 \\
\hline Nasal itching & 29 & 69 \\
\hline Nasal obstruction & 8 & 19 \\
\hline Pale mucosa & 31 & 73.8 \\
\hline Sneezing & 22 & 52.4 \\
\hline
\end{tabular}

Rhinorrhoea (97.6\%), pale mucosa $(73.8 \%)$, nasal itching $(69 \%) \&$ sneezing $(52.4 \%)$ was observed among majority of the patients. 
Table 3: Nasal smeareosniphil countvs AEC

\begin{tabular}{|l|l|l|}
\hline Nasal Smear & AEC Positive & AEC negative \\
\hline positive & 16 & 6 \\
\hline negative & 8 & 12 \\
\hline
\end{tabular}

McNemar Chi square test, $\mathrm{p}<0.05$

Sensitivities of the tests were calculated to be $40 \%$ \& $54 \%$ for nasal smear eosinophil count \& AEC respectively. The difference in sensitivities were found to be significant $(\mathrm{p}<0.05)$.

Out of the 42 patients, with a history of allergic rhinitis 24 had raised AEC and 18 had normal AEC.19 patients had raised nasal smear eosinophil count and the remaining 23 had normal nasal smear eosinophil count. Of the 24 patients with raised AEC, 17 had a raised nasal eosinophil count. Only 2 patients hadraised nasal eosinophil counts with a normal AEC.

The patients were put on Fluticasone propionate nasal spray for one month and the investigations were repeated three days after stopping the treatment.

Out of 24 patients with raised AEC, the AEC of 3(8\%) patients became normal and of the 19 patients with raised nasal smear eosinophil counts, 5(26.3\%) patients' nasal smear eosinophil counts were normalized.The difference in the pre-treatment \& post-treatment values of both the nasal smear eosniphil count $\&$ AEC, irrespective of whether the values were normal or abnormal, were found to be $\operatorname{significant}(\mathrm{p}<0.05)$. AEC reduction was observed in $85.7 \%$ of the patients from the pre-treatment values \& reduction in the nasal smear eosinophil counts were found in $28.6 \%$ of the patients. $88 \%$ of the patients were symptom free after 1 month of treatment and only $12 \%$ had persistence of symptoms even though in these patients, the severity and frequency had reduced.

\section{Discussion}

We compared the sensitivity of the nasal smear eosinophil count with the AEC \& the sensitivity of nasal smear eosinophil count was found to be quite low \& the difference was found to be significant $(\mathrm{p}<0.05)$. Nasal smear eosinophil count is a non-invasive test $\&$ its usefulness in the detection of allergic rhinitis has been shown by various studies.In a study by AkefehAhmediafshar et al[7] the sensitivity was found to be 74\% which was higher than those shown by Miri et al[8]\& Miller et al[9]. But a specificity of $90 \%$ as shown by AkefehAhmediafshar et al [7] was similar to those of the latter two. The specificity of the tests could not be assessed in our study as we did not include subjects who were disease free.The reduction in the eosinophil counts both in the nasal smear eosinophil count\& AEC was found to be significant after treatment with Fluticasone propionate nasal spray. The effectiveness of intranasal corticosteroid therapy in allergic rhinitis has been proven in controlled trials.[10] Treatment with fluticasone nasal spray rendered majority of the patients symptom free which corroborates well with earlier studies.

\section{Conclusion}

Nasal smear eosinophil count was not found to be a significant predictor of allergic rhinitis as AEC. The specificities \&positive predictive values could not be assessed. The number of patients included in the study was limited as many of them were on treatment and had to be excluded. Further evaluation of nasal smear eosinophil count as a diagnostic test in the out-patient setting is necessary.

\section{References}

[1]. Skoner David P: Allergic rhinitis: Definition, epidemiology, pathophysiology, detection and diagnosis. J of Allergy and Clinical

[2]. Immunology volume 108(1) supplement, July 2001; S2-S8.

[3]. James A. Hadley. Otolaryngology for the internist: Evaluation andmanagement of Allergic rhinitis: Medical Clinics OfNorth America- vol 83,Issue 1 (Jan 1999)

[4]. Togias, Askis G: Systemic immunologic and inflammation aspects of Allergic Rhinitis. J of Allergy and Clinical Immunology, vol106 (5)supp, Nov 2000: 5247-5250.

[5]. J. David Osguthorpe. Evolution to Current Otolaryngic Allergy Techniques:Allergy management for the otolaryngologist;

[6]. Otolaryngologic Clinics of NorthAmerica, Vol 31, Number 31, February 1998:1-11.

[7]. Neil Mygind, Robert M Naclerio: Allergic VsNonallergic rhinitis clinical aspectsMunksgaard, Copenhagen, chapter 1, Definition, classification, terminology:11-14.

[8]. Kerr AG. Scott- Brown's otolaryngology Head and Neck SurgeryOxford: Butterworth Heinemann, 1997. Durham SR. Mechanisms and treatment of allergic rhinitis.6th ed.Vol 4:4/6/1-16.

[9]. AkefehAhmadiafshar et al. Nasal eosinophilia as a marker for allergic rhinitis: A controlled study of50 patients. ENT journal. 91(3): 122-124

[10]. Miri S, Farid R, Akbari H, Amin R. Prevalence of allergic rhinitis and nasal smear eosinophilia in 11- to 15 yr-old children in Shiraz.Pediatr Allergy Immunol2006;17(7):519-23.

[11]. Miller RE, Paradise JL, Friday GA, et al. The nasal smear for eosinophils.Its value in children with seasonal allergic rhinitis. AmJ Dis Child 1982;136(11):1009-11.

[12]. A. Parikh et al.Topical corticosteroids in chronic rhinosinusitis:a randomized, double-blind, placebo-controlledtrial using fluticasone propionate aqueous nasalspray. Rhinology, 39, 75-79, 2001 